\title{
EDITORIAL
}

\section{Can inhaled steroids mend a broken heart in chronic obstructive pulmonary disease?}

\author{
D.D. Sin and S.F.P. Man
}

( t has been recognised for several decades that individuals with reduced forced expiratory volume in one second (FEV1) are at increased risk of cardiovascular events, including myocardial infarction, stroke and arrhythmias [1-3]. Since reduced FEV1 and cardiovascular disease often share a common risk factor, cigarette smoking, many had ascribed the above relationship to the confounding effects of smoking. However, a closer examination of the epidemiological data reveals some salient but subtle features that suggest a far more complex reality. First, many of the large epidemiologic studies, which established reduced FEV1 as a risk factor for cardiovascular events, had carefully controlled for the effects of cigarette smoking using sophisticated and well-accepted statistical methods and had excluded at baseline those individuals with overt cardiovascular disease [4]. Secondly, these studies demonstrated that even among life-time nonsmokers, reduced FEV1 was a determinant of cardiovascular disease $[3,5,6]$. Thirdly, the relationship was dose-dependent, such that individuals with the most severe reduction in FEV1 had the highest risk of cardiovascular events, while those with the least amount of impairment in FEV1 had the lowest risk regardless of the smoking status [4]. Taken together, these data indicated that the relationship between reduced FEV1 and cardiovascular disease could not be entirely explained by the effects of cigarette smoking; they implied other causative pathways.

Two decades of epidemiological research have also revealed that the contribution of reduced FEV1 to cardiovascular events is nontrivial. In fact, it is quite large. In one population-based study, Hole et al. [3] showed when the lowest quintile of FEV1 $(<73-75 \%$ of predicted) was compared with the highest quintile, the population-attributable risk for deaths related to ischemic heart disease was $26 \%$ in males and $24 \%$ in females, independent of the burden imposed by cigarette smoking. In other words, in this study, reduced FEV1 may have been responsible for at least $24-26 \%$ of all deaths related to ischemic heart disease. Remarkably, the magnitude of the cardiovascular mortality burden attributed to reduced FEV1 was similar to that imposed by hypercholesterolemia, which had a population attributable risk of $\sim 21-25 \%$ [3].

Other studies have shown that in addition to baseline FEV1, rapid decline in FEV1, a hallmark of chronic obstructive

James Hogg iCAPTURE Center for Cardiovascular and Pulmonary Research, St. Paul's Hospital and Dept of Medicine (Division of Respirology), University of British Columbia, Vancouver, BC, Canada.

CORRESPONDENCE: D.D. Sin, James Hogg iCAPTURE Center for Cardiovascular and Pulmonary Research, St. Paul's Hospital, Room \#368A, 1081 Burrard Street, Vancouver, BC V6Z 1Y6, Canada. Fax: 1 6048069274. E-mail: dsin@mrl.ubc.ca pulmonary disease (COPD), is another risk factor for cardiovascular events. In the Malmo "Men Born in 1914" Study, for instance, the cardiovascular event rate among smokers in the high, middle and low thirds with regard to the decline in FEV1 was 56.0, 41.0 and 22.7 events, respectively, per 1,000 personyrs ( $p$-value for trend $=0.01)$ [7]. In the Baltimore Longitudinal Study of Aging Study, individuals who experienced the most rapid decline in FEV1 over a 16-yr follow-up, were three to five times more likely to die from a cardiac cause of death than those who had the slowest decline in FEV1, after adjustments for age, baseline FEV1, smoking status, hypertension status, body mass index, and mean serum cholesterol level [8]. Even among lifetime nonsmokers, accelerated decline in FEV1 was associated with a 5-10-fold increase in risk for cardiac death, which suggests that the relationship between changes in FEV1 and cardiovascular events occurs independently of the effects of smoking.

In established COPD, the relationship between reduced FEV1 and cardiovascular events is also evident. During the initial 5yr follow-up of the Lung Health Study, which studied 5,887 individuals with mild-to-moderate COPD, cardiovascular event was the principal cause of death in $25 \%$ of the decedents and accounted for $42 \%$ of the first hospitalisations and $48 \%$ of the second hospitalisations [9]. The rate of hospitalisation for lower respiratory tract infection was only one-third of that for cardiovascular illnesses. For every $10 \%$ decrease in FEV1, cardiovascular mortality increased by $28 \%$, and nonfatal coronary events increased by almost $20 \%$, after adjustments for relevant confounders such age, sex, smoking status and treatment assignment [9]. These and several other studies provide solid evidence that pulmonary disorders, such as COPD, contribute significantly to cardiovascular morbidity and mortality and therapies that can mitigate cardiovascular events in COPD may have important implications for the management of COPD patients.

In this issue of the European Respiratory Journal, HUIART et al. [10] report some promising data on the use of inhaled corticosteroids to possibly lower the incidence of myocardial infarction in COPD. They studied 371 cases and 1,864 control subjects in 1990-1997 and found that users of inhaled corticosteroids had a nonsignificant $18 \%$ reduction in the risk for acute myocardial infarction. Most of the beneficial signal came from a subgroup that used on average 50-200 ug per day of inhaled beclomethasone or equivalent. Remarkably, this subgroup experienced a $32 \%$ relative reduction in the risk for acute myocardial infarction $(\mathrm{p}<0.05)$. However, this study failed to find a dose-response relationship, as one would hope to see in a biological system [11]. The obvious strengths of this 
study are the large sample size, the population-based approach to patient sampling and the likely complete ascertainment of acute myocardial infarction events in the cohort. However, this study was not a randomised controlled trial and contains all the major shortcomings of observational studies [12], which invariably lead to the question, "are the results valid or are they confounded?". Additionally, the databases used by the authors did not contain FEV1 data or smoking history. In lieu of this, the authors used receipt of anti-COPD medications to define COPD, making the case definition of COPD problematic. For these and other reasons, observational studies such as this one cannot be considered definitive and should not cause us to change our therapeutic approach in managing patients with COPD [12].

The real merit of this study is that it challenges us to consider the effects of inhaled corticosteroids beyond the traditional limits of the pulmonary system. Corticosteroids are potent but nonspecific anti-inflammatory agents. As such, when given as an aerosol, they broadly down-regulate the inflammatory process in the airways [13]. If the nidus of the systemic inflammation, which is commonly observed in patients with established COPD [14], is airway inflammation, it is possible that by down-regulating airway inflammation, systemic inflammation may also be mitigated by inhaled corticosteroids. Consistent with this notion, PINTO-PLATA et al. [15] reported that circulating C-reactive protein (CRP) levels, a measure for systemic inflammation, were $\sim 20 \%$ lower among patients with COPD who used inhaled corticosteroids, compared with those who did not. Similarly, we found among 41 patients with COPD that withdrawal of inhaled corticosteroids increased serum CRP levels by $\sim 71 \%$, while 2 weeks on inhaled fluticasone reduced CRP levels by $\sim 50 \%$ [16]. Inhaled fluticasone also significantly reduced serum interleukin-6 levels by $26 \%$. If these initial observations can be corroborated by other studies, down-regulation of systemic inflammation may be one potential pathway by which inhaled corticosteroids can confer beneficial effects on the cardiovascular system. This is plausible because systemic inflammation is an important cofactor in the genesis of atherosclerosis and plaque rupture [17] and certain therapies that attenuate systemic inflammation improve cardiovascular outcomes [18, 19].

However, before accepting the notion that inhaled corticosteroids can "mend a broken heart", more animal and clinical studies are needed to better understand the mechanistic and epidemiologic link between steroid therapy and cardiovascular disease in chronic obstructive pulmonary disease. Observational studies such as this one by HUIART et al. [10] are best used to generate new hypotheses. To this end, the current study has further added to the call for more research in this and other new and exciting areas of research in chronic obstructive pulmonary disease.

\section{REFERENCES}

1 Engstrom G, Wollmer P, Hedblad B, Juul-Moller S, Valind S, Janzon L. Occurrence and prognostic significance of ventricular arrhythmia is related to pulmonary function: a study from "men born in 1914," Malmo, Sweden. Circulation 2001; 103: 3086-3091.

2 Truelsen T, Prescott E, Lange P, Schnohr P, Boysen G. Lung function and risk of fatal and non-fatal stroke. The
Copenhagen City Heart Study. Int J Epidemiol 2001; 30: 145-151.

3 Hole DJ, Watt GC, Davey-Smith G, Hart CL, Gillis CR, Hawthorne VM. Impaired lung function and mortality risk in men and women: findings from the Renfrew and Paisley prospective population study. BMJ 1996; 313: 711-715.

4 Sin DD, Wu L, Man SFP. The relationship between reduced lung function and cardiovascular mortality: a populationbased study and a systematic review of the literature. Chest 2005; (In press).

5 Strachan DP. Ventilatory function, height, and mortality among lifelong non-smokers. J Epidemiol Community Health 1992; 46: 66-70.

6 Curb JD, Marcus EB, Reed DM, MacLean C, Yano K. Smoking, pulmonary function, and mortality. Ann Epidemiol 1990; 1: 25-32.

7 Engstrom G, Hedblad B, Janzon L, Valind S. Respiratory decline in smokers and ex-smokers- an independent risk factor for cardiovascular disease and death. J Cardiovasc Risk 2000; 7: 267-272.

8 Tockman MS, Pearson JD, Fleg JL, et al. Rapid decline in FEV1. A new risk factor for coronary heart disease mortality. Am J Respir Crit Care Med 1995; 151: 390-398.

9 Anthonisen NR, Connett JE, Enright PL, Manfreda J. Lung Health Study Research Group. Hospitalizations and mortality in the Lung Health Study. Am J Respir Crit Care Med 2002; 166: 333-339.

10 Huiart L, Ernst P, Ranouil X, Suissa S. Low-dose inhaled corticosteroids and the risk of myocardial infarction in COPD. Eur Respir J 2005; 25: 634-639.

11 Deeks JJ, Dinnes J, D'Amico R, et al. Evaluating nonrandomised intervention studies. Health Technol Assess 2003; 7: 1-173.

12 Grimes DA, Schulz KF. Bias and causal associations in observational research. Lancet 2002; 359: 248-252.

13 Faul JL, Leonard CT, Burke CM, Tormey VJ, Poulter LW. Fluticasone propionate induced alterations to lung function and the immunopathology of asthma over time. Thorax 1998; 53: 753-761.

14 Gan WQ, Man SF, Senthilselvan A, Sin DD. Association between chronic obstructive pulmonary disease and systemic inflammation: a systematic review and a metaanalysis. Thorax 2004; 59: 574-580.

15 Pinto-Plata V, Muellerova $\mathrm{H}$, Toso J, et al. C-reactive protein is elevated in patients with COPD but not in smoker and non-smoker controls. CRP is influenced by the use of MDI corticosteroids. Am J Respir Crit Care Med 2004; 169: Suppl. A839.

16 Sin DD, Lacy P, York E, Man SF. Effects of fluticasone on systemic markers of inflammation in chronic obstructive pulmonary disease. Am J Respir Crit Care Med 2004; 170: 760-765.

17 Ross R. Atherosclerosis- an inflammatory disease. N Engl J Med 1999; 340: 115-126.

18 Ridker PM, Cannon CP, Morrow D, et al. Pravastatin or Atorvastatin Evaluation and Infection TherapyThrombolysis in Myocardial Infarction 22 (PROVE IT-TIMI 22) Investigators: C-reactive protein levels and outcomes after statin therapy. N Engl J Med 2005; 352: 20-28.

19 Choi HK, Hernan MA, Seeger JD, Robins JM, Wolfe F. Methotrexate and mortality in patients with rheumatoid arthritis: a prospective study. Lancet 2002; 359: 1173-1177. 